\title{
INHALTSVERZEICHNIS der Nummern 1-6 24. Jahrgang (1997)
}

\section{Artikel}

Blei, Dagmar: Deutsch als Fremdsprache in der DDR. Ein Beitrag zur Fach- und Wissenschaftsgeschichte

Bode, Christian: Deutsch als Fremdsprache für ausländische Studierende und Wissenschaftler

Bosch, Gloria: Sprachenpolitik und Fremdsprachenunterricht im vereinten Europa

Ehlich, Konrad: Von der Attraktivität der Lehrangebote für »Deutsch als fremde Wissenschaftssprache $«$. Wissenschaftspolitische Voraussetzungen und didaktische Konsequenzen

López Barrios, Mario: Wörterbuch und Textproduktion

Roggausch, Werner: Deutschlehrerausbildung: Thesen zur Curriculum-Planung

Roggausch, Werner: Kulturkontrast und Hermeneutik. Einige Notizen zur Begriffsbildung in den Geisteswissenschaften

Schwerdtfeger, Inge Christine: Der Unterricht Deutsch als Fremdsprache: Auf der Suche nach den verlorenen Emotionen

Siebke, Martina: Etymologisierung im Fremdsprachenunterricht

Stephani, Christiane: Über das Partizipialattribut oder: Über die Tatsache, daß Hänschen die Schuhe kennen muß, und daß Hans wissen muß, daß ein rechter Schuh kein linker Schuh ist - außer bei groben Filzpantoffeln

Unterbäumen, Enrique Huelva: Abstraktionsgrad der grammatikalischen Beschreibung und Aufbau der Lernergrammatik

Wolff, Armin; Eggers, Dietrich; Ehnert, Rolf; Kirsch, Klaus: Deutsch als Fremdsprache und der Studienstandort Deutschland. Entwicklungslinien eines Faches aus der Sicht (s)eines Verbandes

$6,780-795$

$6,751-756$

$4,459-469$

$6,757-770$

$1,3-19$

$4,470-479$

$6,796-807$

$5,587-606$

$1,20-24$

$6,771-779$

$1,25-50$

$5,559-586$

$4,493-498$

$1,58-68$

Hinrichs, Beatrix: Zur Problematik des DaF-Unterrichts an Deutschen Schulen im Ausland. Dargestellt am Beispiel einer deutsch-peruanischen Begegnungsschule

Majari, Chris F.: Ein Blick über den Zaun: Eindrücke aus den USA vom Fremdsprachenlernen mit Hypertext und Hypermedia

Saengaramruang, Wanna: Aufgabenstellung in einem thailandspezifischen Deutschlehrwerk im Hochschulbereich

Tallowitz, Ulrike und Fandrych, Christian: Entwicklung eines Fernstudiengangs für DaF-Lehrer an mexikanischen Universitäten

Żurek, Jolanta: Polnische Stereotypenbilder über Deutschland und Deutsche

$6,808-817$

$5,607-620$

$1,51-57$

$4,480-492$

$5,625-639$

Info DaF 24, 6 (1997), 854-866 


\section{Didaktik DaF/Aus der Praxis}

Barner, Axel: Das Eigene und das Fremde sehen. Der »Stadtführer Bukarest«als Projektarbeit mit Studenten der Bukarester >Politehnica<

$5,656-660$

Berndt Annette: Fremdsprachenlernen im höheren Erwachsenenalter. Ansätze zu einer Sprachgeragogik

Bolten, Jürgen: Das HRK-Zertifikat »Fachsprache Wirtschaft «. Richtlinien, Ausbildung und Prüfungspraxis

Bräsel, Sylvia: Blickwechsel - Überlegungen zur Landeskunde in einer sich verändernden Welt. Versuch einer Annäherung im ostasiatischen Kontext

Drewnowska-Vargáné, Ewa: Textfunktion und Textstruktur als Grundlagen für die Entwicklung der Textkompetenz

Kaikkonen, Pauli: Fremdverstehen durch schulischen Fremdsprachenunterricht. Einige Aspekte zum interkulturellen Lernen

Kim, Hi-Youl: Die Problematik der Sprachpraxis im Fach Germanistik in Korea

Meuter, Jörg: TURBO, ein Videomagazin für Jugendliche. Zum Einsatz von landeskundlichen Videobeiträgen im DaF-Unterricht

Reinbothe, Roswitha: Landeskunde in der Deutschlehrerausbildung

Schlabach, Joachim: Erfahrungen und sprachliche Probleme beim Auslandsstudium: Finnische Wirtschaftsstudierende in Deutschland und Österreich

Schütz, Sonja: Kabarett-Texte als alternative Textsorte für den DaF-Unterricht. $\mathrm{Zu}$ den didaktisch methodischen Potentialen einer bisher vernachlässigten Textsorte

Topalova, Antoanita: »Falsche Freunde« im Klassenzimmer. Fehler beim Internationalismen-Gebrauch deutschlernender Bulgaren

Wehage, Franz-Joseph: Einsatz von Videos im DaF-Unterricht aus US-amerikanischer Sicht

$1,69-77$

$4,530-541$

$1,99-113$

$4,514-522$

$1,78-86$

$5,640-651$

$5,652-655$

$4,499-513$

$6,818-828$

$1,87-98$

$5,661-666$

$4,523-529$

\section{Berichte}

Berichte über die 25. Jahrestagung Deutsch als Fremdsprache an der Johannes Gutenberg-Universität Mainz vom 22. bis 24. Mai 1997

Themenschwerpunkt 1: Emotion und Kognition beim Fremdsprachenlernen Gegensätze und Gemeinsamkeiten (Frank G. Königs, Gabriele Neuf-Münkel, Anke Stöver-Blahak)

Themenschwerpunkt 2: Lernen mit neuen Medien (Christiane Braun, Rüdiger Schreiber, Klaus Vorderwülbecke)

Themenschwerpunkt 3: Deutsch als Fremdsprache und die Attraktivität des Lern- und Studienstandortes Deutschland (Hans-Georg Albers, Brigitte Krefting, Bernd Wintermann)

Forum Deutsch als Fremdsprache (Renate Henkenborg-Schröder)

Koeppel, Rolf: Die chinesische Germanistik im 21. Jahrhundert. Bericht von einer Tagung in Qingdao, VR China, vom 26. bis 30.07.1997

Mehrsprachigkeit in Europa - woher kommen die Fremdsprachenlehrer? Erklärung der 17. Frühjahrskonferenz zur Erforschung des Fremdsprachenunterrichts zur Fremdsprachenlehrerausbildung und zum Fremdsprachenunterricht an Hochschulen

Oybiner Erklärung zu Rolle und Stellenwert der Studienkollegs für die Attraktivität des Studienstandorts Deutschland

Pressemitteilung der Arbeitsgemeinschaften der Studienkollegs für ausländische Studierende in der Bundesrepublik Deutschland 
Rügener Erklärung zu Rolle und Stellenwert der Studienkollegs für die Attraktivität des Studienstandorts Deutschland

Wiesmann, Bettina: Ergebnisse der Fachtagung »Umsetzung der neuen DSHOrdnung in die Prüfungspraxis « vom 28.-30.11.1996 in Regensburg

Wiesmann, Bettina: Ergebnisse der FaDaF-Fachtagung »Die DSH in der Praxis: Prüfungsvorbereitung « vom 2. bis 4. Oktober 1997 in Regensburg

Zimmer, Thomas: »Deutsch als Fremdsprache in Korea - Tendenzen und Prognosen«. Bericht über eine Tagung vom 15.-17.11.1996 in Yangpeyong

$4,542-544$

\section{Aus der Arbeit des FaDaF}

Berichte über die 25. Jahrestagung Deutsch als Fremdsprache an der Johannes Gutenberg-Universität Mainz vom 22. bis 24. Mai 1997

Protokoll der Mitgliederversammlung des Fachverbandes Deutsch als Fremdsprache (FaDaF) im Rahmen der 25. Jahrestagung DaF (Brigitte Krefting)

Rechenschaftsbericht des Vorstandes des Fachverbandes Deutsch als Fremdsprache für das Geschäftsjahr 1996/97 (Bernd Wintermann)

Vorankündigung der 26. Jahrestagung Deutsch als Fremdsprache des Fachverbandes Deutsch als Fremdsprache vom 4. bis 6. Juni 1998 an der FriedrichSchiller-Universität Jena

\section{Auswahlbibliographie/Rezensionen/Eingegangene Literatur}

Auswahlbibliographie von Neuerscheinungen für das Fach Deutsch als Fremdsprache 1996; zusammengestellt von Dietrich Eggers und Evelyn MüllerKüppers, unter Mitarbeit von Dorothee Schwarck und Arno Sandner

Albers, Hans-Georg (Hrsg.): Fort- und Weiterbildung von Lehrkräften für Deutsch als Fremdsprache. Vorträge und Materialien der Fachtagung in Bad Emstal-Sand 1993 (Rüdiger Riechert)

Arnold, Heinz Ludwig (Hrsg): Ansichten und Auskünfte zur deutschen Literatur nach 1945 (Henrik Engel)

Auernheimer, Georg: Einführung in die interkulturelle Erziehung (Regina Richter)

Barz, Irmhild; Schröder Marianne (Hrsg.): Das Lernerwörterbuch Deutsch als Fremdsprache in der Diskussion (Lutz Köster)

Baurmann, Jürgen; Weingarten, Rüdiger (Hrsg.): Schreiben. Prozesse, Prozeduren, Produkte (Eva-Maria Willkop)

Bausch, Karl-Richard; Christ, Herbert; Krumm, Hans-Jürgen (Hrsg.): Handbuch Fremdsprachenunterricht. 3. überarbeitete und erweiterte Auflage (Karl Esselborn)

Bausch, Karl-Richard; Christ, Herbert; Königs, Frank G.; Krumm, Hans-Jürgen (Hrsg.): Erwerb und Vermittlung von Wortschatz im Fremdsprachenunterricht. Arbeitspapiere der 15. Frühjahrskonferenz zur Erforschung des Fremdsprachenunterrichts (Oliver Bayerlein)

Beck, Erwin; Guldimann, Titus; Zutavern, Michael (Hrsg.): Eigenständig lernen (Claudia Hilker)

Beedham, Christopher: German Linguistics. An Introduction (Ralph Hartmann) 
Berg, Hans Christoph; Schulze, Theodor: Lehrkunst. Lehrbuch der Didaktik (Daniela Hartmann)

Besançon, Anne: La compréhension de l'allemand. Langue et culture (Walter Schleyer)

$2 / 3,200-204$

$5,699-704$

Bittner, Andreas: Starke sschwache Verben - sschwache`starke Verben. Deutsche Verbflexion und Natürlichkeit (Jörg Riecke)

Bollenbeck, Georg: Bildung und Kultur: Glanz und Elend eines deutschen Deutungsmusters (Arpad A. Sölter)

Brand, Linda; Kresin-Murakami, Jutta; Pechatscheck, Karl: Die Schöne ist angekommen. Ein Grammatikkrimi (Maria Yendell)

Bredella, Lothar; Christ, Herbert (Hrsg.): Didaktik des Fremdverstehens (Claus Altmayer)

Brenner, Gerd: Besser in allen Fächern. Sachtexte verstehen und verfassen. Oberstufe (Hans-Otto Rößer)

Brockhaus, Wiebke: Final Devoicing in the Phonology of German (Michael Groß)

Bugdahl, Volker: Kreatives Problemlösen im Unterricht (Wim Waumans)

Bünting, Karl-Dieter: Schreiben im Studium. Ein Trainingsprogramm (Inge Wüst)

Butzkamm, Wolfgang: Unterrichtssprache Deutsch. Wörter und Wendungen für Lehrer und Schüler (Karl-Walter Florin)

Calvin, William H.; Ojemann, George A.: Einsicht ins Gehirn. Wie Denken und Sprache entstehen (Holger Nord)

Chen, Shing-lung: Pragmatik des Passivs in chemischer Fachkommunikation: Empirische Analysen von Labordiskursen, Versuchsanleitungen, Vorlesungen und Lehrwerken (Bettina Wiesmann)

Claussen, Claus; Merkelbach, Valentin: Erzählwerkstatt (Manfred Niedballa)

Conlin, Christine: Unternehmen Deutsch. Ein berufsorientierter Kurs für die Grundstufe. Lehrbuch (einsprachig), Lehrbuch (englische Ausgabe), Arbeitsbuch (einsprachig), 2 Cassetten, Lehrerhandreichung (Christine Stief)

Crystal, David: Die Cambridge Enzyklopädie der Sprache. Studienausgabe (Britt-Marie Schuster)

Czyżewski, Marek; Gülich, Elisabeth; Hausendorf, Heiko; Kastner, Maria (Hrsg.): Nationale Selbst- und Fremdbilder im Gespräch. Kommunikative Prozesse nach der Wiedervereinigung Deutschlands und dem Systemwandel in Ostmitteleuropa (Claus Altmayer)

Danneberg, Lutz; Vollhardt, Friedrich (Hrsg.): Wie international ist die Literaturwissenschaft? Methoden- und Theoriediskussion in den Literaturwissenschaften: kulturelle Besonderheiten und interkultureller Austausch am Beispiel des Interpretationsproblems (1950-1990) (Gertrud Maria Rösch)

Deitering, Franz-G.: Selbstgesteuertes Lernen (Karl-Walter Florin)

Demandt, Alexander (Hrsg.): Mit Fremden leben. Eine Kulturgeschichte von der Antike bis zur Gegenwart (Sylvia Bräsel)

Dittmar, Norbert; Rost-Roth, Martina (Hrsg.): Deutsch als Zweit- und Fremdsprache. Methoden und Perspektiven einer akademischen Disziplin (Matthias Grünewald)

Effenberger, Sabine: Fremdsprachenunterricht in den USA. Bedingungen und Strukturen an den Hochschulen der USA unter besonderer Berücksichtigung des Faches Deutsch (Anneliese Stein-Meintker)

Ehlich, Konrad (Hrsg.): Kindliche Sprachentwicklung. Konzepte und Empirie (Andrea Jahnel)

Engler, Bernd; Müller, Kurt (Hrsg.): Exempla. Studien zur Bedeutung und Funktion exemplarischen Erzählens (Harald Tanzer)

Eschenbach, Carola: Zählangaben - Maßangaben. Bedeutung und konzeptuelle Interpretation von Numeralia (Hardarik Blühdorn)

$2 / 3,205-208$

$2 / 3,209-211$

$2 / 3,212-213$

$2 / 3,213-215$

$2 / 3,333-336$

$2 / 3,216-218$

$2 / 3,218-222$

$2 / 3,223-224$

$2 / 3,224-225$

$2 / 3,225-227$

$2 / 3,227-229$

2/3, 230-231

$2 / 3,231-233$

$2 / 3,233-236$

$2 / 3,237-241$

$2 / 3,241-246$

2/3, 246-248

5, 704-706

$2 / 3,249-250$

5, 706-709

2/3, 251-255

2/3, 255-257

$2 / 3,257-262$ 
Fiehler, Reinhard; Metzing, Dieter (Hrsg.): Untersuchungen zur Kommunikationsstruktur (Monika Dannerer)

Firnhaber-Sensen, Ulrike; Schmidt, Gabriele: Deutsch im Krankenhaus. Berufssprache für ausländische Pflegekräfte. Lehr- und Arbeitsbuch. Audiocassette. Lehrerhandreichung (Harald Tanzer)

Fohrmann, Jürgen; Müller, Harro (Hrsg.): Literaturwissenschaft (Peter Jaumann)

Frey, Evelyn: Kursbuch Phonetik. Lehr- und Übungsbuch. Package: Lehr- und Übungsbuch und 2 Cassetten (Ursula Saarbeck)

Gauger, Hans-Martin: Über Sprache und Stil (Gertrud Maria Rösch)

Giacalone Ramat, Anna; Crocco Galèas, Grazia (Hrsg.): From Pragmatics to Syntax. Modality in Second Language Acquisition (Andrea Jahnel)

Gruber, Helmut: Streitgespräche. Zur Pragmatik einer Diskursform (Bernd Latour)

Gutknecht, Christoph: Lauter Böhmische Dörfer. Wie die Wörter zu ihrer Bedeutung kamen (Inula Wolter)

Hahn, Hans-Joachim: German Thought and Culture. From the Holy Roman Empire to the Present Day (Arpad A. Sölter)

Hakkarainen, Heikki J.: Phonetik des Deutschen (Michael Groß)

Hall, Karin; Scheiner, Barbara: Übungsgrammatik Deutsch als Fremdsprache für Fortgeschrittene. Lösungsschlüssel (Gerhard Bickes)

Handwerker, Brigitte (Hrsg.): Fremde Sprache Deutsch: Grammatische Beschreibung - Erwerbsverläufe - Lehrmethodik (Roswitha Althoff)

Hasebrook, Joachim: Multimedia-Psychologie. Eine neue Perspektive menschlicher Kommunikation (Oliver Bayerlein)

Häublein, Gernot; Müller, Martin; Rusch, Paul; Scherling, Theo; Wertenschlag, Lukas: Memo. Wortschatz- und Fertigkeitstraining zum Zertifikat Deutsch als Fremdsprache. Lehr- und Übungsbuch. Lernwortschatz Deutsch-Deutsch. Deutsch-Englisch. Deutsch-Französisch. Deutsch-Italienisch. Deutsch-Spanisch. Audiocassette (Regina Richter)

Heinze-Prause, Roswitha; Heinze, Thomas: Kulturwissenschaftliche Hermeneutik. Fallrekonstruktionen der Kunst-, Medien- und Massenkultur (Claus Altmayer)

Helbig, Jörg: Intertextualität und Markierung. Untersuchungen zur Systematik und Funktion der Signalisierung von Intertextualität (Beate Wolfsteiner)

Henrici, Gert; Zöfgen, Ekkehard (Hrsg.): Kontrastivität und kontrastives Lernen (Eva-Maria Willkop)

Herzmann, Herbert (Hrsg.): Literaturkritik und erzählerische Praxis. Deutschsprachige Erzähler der Gegenwart. Tagungsakten des internationalen Symposiums University College. Dublin, 14.-16. Februar 1993 (Sibille Tröml)

Heusinger, Siegfried: Pragmalinguistik, Texterzeugung, Textanalyse; Stilgestaltung und Stilwirkungen in der sprachlichen Kommunikation. Ein Lehr- und Übungsbuch (Daniela Hartmann)

Heyne, Isolde: Jenny und Jojo (Ilse Heinecke)

Hönig, Hans G.: Konstruktives Übersetzen (Walter Schleyer)

Hof, Renate: Die Grammatik der Geschlechter. Gender als Analysekategorie der Literaturwissenschaft (Gertrud Maria Rösch)

Hundt, Markus: Modellbildung in der Wirtschaftssprache. Zur Geschichte der Institutionen- und Theoriefachsprachen der Wirtschaft (Ewald Reuter)

Inglehart, Ronald: Kultureller Umbruch. Wertewandel in der westlichen Welt (Marlis Wilde-Stockmeyer)

Inhetveen, Rüdiger; Kötter, Rudolf (Hrsg.): Betrachten, Beobachten, Beschreiben: Beschreibungen in Kultur- und Naturwissenschaften (Carola Niedenthal) 
Jakobs, Eva-Maria; Knorr, Dagmar; Molitor-Lübbert, Sylvie (Hrsg.): Wissenschaftliche Textproduktion - Mit und ohne Computer (Gunther Dietz)

$2 / 3,319-323$

Kegelmann, René: »An den Grenzen des Nichts, dieser Sprache ...« Zur Situation rumänien-deutscher Literatur der achtziger Jahre in der Bundesrepublik Deutschland (Karl Esselborn)

Keller, Rudi: Zeichentheorie. Zu einer Theorie semiotischen Wissens (Enrique Huelva Unterbäumen)

Kelz, Heinrich P.: Deutsche Aussprache. Praktisches Lehrbuch zur Ausspracheschulung für den Unterricht in Deutsch als Fremdsprache (Ursula Saarbeck)

Kessler, Michael; Wertheimer, Jürgen (Hrsg.): Multikulturalität. Tendenzen Probleme - Perspektiven im europäischen und internationalen Horizont (Henrik Engel)

Klute, Wilfried: Besser in Deutsch. Sachtexte analysieren. Oberstufe (Hans-Otto Rößer)

Kniffka, Hannes: Elements of Culture-Contrastive Linguistics. Elemente einer kulturkontrastiven Linguistik (Holger Nord)

Kraft, Helga: Ein Haus aus Sprache. Dramatikerinnen und das andere Theater (Astrid Volmer)

Krusche, Dietrich: Leseerfahrung und Lesergespräch (Irmgard Honnef-Becker)

Kühn, Peter: Mehrfachadressierung. Untersuchungen zur adressatenspezifischen Polyvalenz sprachlichen Handelns (Sigrid Luchtenberg)

Kühn, Peter (Hrsg.): Hörverstehen im Unterricht DaF. Theoretische Fundierung und unterrichtliche Praxis (Inge Wüst)

Langer, Gudrun: Textkohärenz und Textspezifität. Textgrammatische Untersuchungen zu den Gebrauchstextsorten Klappentext, Patienteninformation, Garantieerklärung und Kochrezept (Gunther Dietz)

Langner, Heidemarie C.: Die Schreibung englischer Entlehnungen im Deutschen. Eine Untersuchung zur Orthographie von Anglizismen in den letzten hundert Jahren, dargestellt an Hand des Duden (Michael Groß)

Latzel, Sigbert: Lernschwierigkeiten mit deutschen Synonymen. Teil I: Eine Analyse von 30 Gruppen sinnverwandter stammgleicher Verben. Teil II: Übungen zu 30 Verbgruppen (János Mohácsi/Zsuzsanna Antal)

Leisi, Ernst: Streiflichter. Unzeitgemäße Essays zur Kultur, Sprache und Literatur (Henrik Engel)

Liedtke, Frank (Hrsg.): Implikaturen. Grammatische und pragmatische Analysen (Sigrid Luchtenberg)

Litters, Ulrike: Interkulturelle Kommunikation aus fremdsprachendidaktischer Perspektive. Konzeption eines zielgruppenspezifischen Kommunikationstrainings für deutsche und französische Manager (Gabriele Padberg)

Lodewick, Klaus: Gegensätze. Ein Programm für die Mittelstufe Deutsch als Fremdsprache. Textbuch. Arbeitsbuch. LehrerInnenhandbuch. 2 Audiocassetten. Foliensatz. Tests und Spiele (Lutz Götze)

Luscher, Renate: Deutschland nach der Wende. Daten, Texte, Aufgaben für Deutsch als Fremdsprache (Kirsten Bohle)

Luscher, Renate: deutsch rapid. A German Self-Study Course for Beginners. Cassettenkurs Deutsch-Englisch (Andrea Wagner)

Lutzeier, Peter Rolf: Lexikologie. Ein Arbeitsbuch (Ralph Hartmann)

Macaire, Dominique; Nicolas, Gerd: Wirtschaftsdeutsch für Anfänger. Grundstufe. Lehr- und Arbeitsbuch. 2 Cassetten (Monique Ansorge)

Mahle, Walter A. (Hrsg.): Deutschland in der internationalen Kommunikation (Inge Mohr)

Mavrodieva, Ljubov; Sretkova, Anna; Stankulowa, Krystina K.: Deutschland und der deutsche Alltag. Ein Landeskundeprogramm für bulgarische Schüler (Rainer Bettermann)

$2 / 3,323-326$

5, 716-719

$2 / 3,327-328$

$2 / 3,328-333$

2/3, 333-336

2/3, 336-339

$5,719-722$

2/3, 339-341

$2 / 3,341-343$

$2 / 3,343-345$

$2 / 3,345-349$

$2 / 3,349-351$

5, 722-725

$2 / 3,351-352$

2/3, 353-355

$2 / 3,355-356$

$2 / 3,356-358$

$2 / 3,358-360$

$2 / 3,360-362$

$2 / 3,362-363$

$2 / 3,364-365$

2/3, 365-367

5, 689-694 
Moser, Heinz: Einführung in die Medienpädagogik. Aufwachsen im Medienzeitalter (Thomas Bleicher)

Müller, Siegfried; Otto, Hans-Uwe; Otto, Ulrich (Hrsg.): Fremde und Andere in Deutschland. Nachdenken über das Einverleiben, Einebnen, Ausgrenzen (Karl Esselborn)

Münch, Winfried: Individuum und Gruppe in der Weiterbildung. Psychologische Grundlagen für die Praxis in Seminaren, Kursen und Training (Rüdiger Riechert)

Otto, Wolf-Dieter: Wissenschaftskultur und Fremde. Auswärtige Kulturarbeit als Beitrag zur interkulturellen Bildung. Auch eine pädagogische Reflexion asiatischer Lehrjahre in Korea (Sylvia Bräsel)

Pabst-Weinschenk, Marita: Reden im Studium. Ein Trainingsprogramm (Katharina Mikova)

Papp, Edgar: Taschenbuch Literaturwissenschaft. Ein Studienbegleiter für Germanisten (Stefani Sonntag)

Pflanz, Manfried Hermann: Moderner Roman, Satire und Kunstmärchen im Unterricht. Anregungen zur Unterrichtsgestaltung für Deutschlehrer am Gymnasium (Beate Wolfsteiner)

Plumpe, Gerhard: Epochen moderner Literatur. Ein systemtheoretischer Entwurf (Sibille Tröml)

Plumpe, Gerhard; Werber, Niels (Hrsg.): Beobachtungen der Literatur. Aspekte einer polykontexturalen Literaturwissenschaft (Henrik Engel)

Pohl, Inge; Ehrhardt, Horst (Hrsg.): Wort und Wortschatz, Beiträge zur Lexikologie (Karl-Walter Florin)

Punkki-Roscher, Marja: Nominalstil in populärwissenschaftlichen Texten. Zur Syntax und Semantik der komplexen Nominalphrasen (Zenon Weigt)

Rampillon, Ute: Lernen leichter machen. Deutsch als Fremdsprache (Claudia Hilker)

Reimann, Monika: Grundstufen-Grammatik für Deutsch als Fremdsprache. Erklärungen und Übungen. Lösungsschlüssel (Rüdiger Heßling)

Reuter, Ewald (Hrsg.): FINLANCE. A Finnish Journal of Applied Linguistics. Volume XV: Fremdsprachliches Textverstehen (Anita Dreischer)

Rösch, Heidi: Interkulturell unterrichten mit Gedichten. Zur Didaktik der Migrationslyrik (Karin Yesilada)

Rost-Roth, Martina (unter Mitarbeit von Oliver Lechlmair): Sprachenlernen im direkten Kontakt. Autonomes Tandem in Südtirol. Eine Fallstudie (Karin Aguado)

Sager, Sven F.: Verbales Verhalten. Eine semiotische Studie zur linguistischen Ethologie (Bernd Latour)

Samel, Ingrid: Einführung in die Feministische Sprachwissenschaft (Andrea Jahnel)

Schanda, Franz: Computer-Lernprogramme. Wie damit gelernt wird. Wie sie entwickelt werden. Was sie im Unternehmen leisten (Haymo Mitschian)

Schanen, François: Grammatik Deutsch als Fremdsprache (Anneliese SteinMeintker)

Schewe, Manfred: Fremdsprache inszenieren. Zur Fundierung einer dramapädagogischen Lehr- und Lernpraxis (Peter Jankowsky)

Schewe, Manfred; Wilms, Heinz u.a.: Texte lesen und inszenieren. Alfred Andersch: Sansibar oder der letzte Grund (Jutta Seifert)

Schlag, Bernhard: Lern- und Leistungsmotivation (leva Vinkalne)

Schlipper, Annette: Julias Traum (Ilse Heinecke)

Schmidt-Veitner, Claudia; Wieland, Regina: Grammatik aus Texten. Übungen zu Adjektiven, Nomen und Verben für die Mittelstufe. Lösungsschlüssel (Christian Krekeler)

$2 / 3,367-369$

$2 / 3,369-372$

$2 / 3,372-373$

$5,725-728$

$2 / 3,374-375$

$2 / 3,375-376$

2/3, 377-378

2/3, 378-381

2/3, 381-384

2/3, 384-385

5, 728-731

2/3, 385-386

2/3, 386-388

2/3, 388-391

5, 731-733

5, 733-738

$2 / 3,392-393$

2/3, 393-397

2/3, 397-399

$2 / 3,400-403$

2/3, 403-405

$2 / 3,405-406$

$2 / 3,407-408$

2/3, 408

2/3, 408-411 
Schmitter, Peter (Hrsg.): Sprachtheorien der Neuzeit II. Von der Grammaire de Port-Royal (1660) zur Konstitution moderner linguistischer Disziplinen (Detlef Otto)

$2 / 3,411-414$

Schoop, Eric; Witt, Ralf; Glowalla, Ulrich (Hrsg.): Hypermedia in der Aus- und Weiterbildung. Dresdner Symposium zum computerunterstützten Lernen (Haymo Mitschian)

Sion, Christopher (Hrsg.): 88 Unterrichtsrezepte. Eine Sammlung interaktiver Übungsideen (Daniela Hartmann)

Spiegel, Carmen: Streit. Eine linguistische Untersuchung verbaler Interaktionen in alltäglichen Zusammenhängen (Bernd Latour)

Spillner, Bernd (Hrsg.) Sprache: Verstehen und Verständlichkeit. Kongreßbeiträge zur 25. Jahrestagung der Gesellschaft für Angewandte Linguistik GAL e. V. (Gotthard Wildegans)

Stöpfgeshoff, Dieter; Schmiedecke, Manuela; Kantoreitis, Peter: Einblicke und Ansichten (Georg Schmidt)

Teske, Gudrun: Dosenschiffchen (Ilse Heinecke)

Themen neu 2: Aufderstraße, Hartmut; Bock, Heiko; Müller, Jutta: Lehrerhandbuch Teil B. Vorlagen, Hinweise zu Grammatik und Landeskunde, Tests (Matthias Grünewald)

Ur, Penny; Wright, Andrew: 111 Kurzrezepte Deutsch als Fremdsprache (Wim Waumans)

Vögeding, Joachim: »Wenn in einen gesättigten Wasser Kochsalz gibt ...« Zur Lernbarkeit von Naturwissenschaften in einer Fremdsprache - am Beispiel eines deutschsprachigen Chemieunterrichts in der Türkei (Istanbul Lisesi) (Gotthard Wildegans)

Volkmer, Ingrid; Lauffer, Jürgen (Hrsg.) Kommunikative Kompetenz in einer sich verändernden Medienwelt (Inge Mohr)

Volmert, Johannes (Hrsg.): Grundkurs Sprachwissenschaft: Eine Einführung in die Sprachwissenschaft für Lehramtsstudiengänge (Irene Doval)

Vorderwülbecke, Anne: Gespräche mit Lunija. Ein Hörprogram mit Musik für die Grundstufe (Inge Wüst)

Wicke, Rainer E.: Handeln und Sprechen im Deutschunterricht. Spielerische Sprachaktivitäten (Rosemarie Freyer-Wojnikowa)

Wicke, Mariele; Wicke, Rainer: Ich schreibe über mich (Rosemarie FreyerWojnikowa)

Wierlacher, Alois; Eggers, Dietrich; Engel, Ulrich; Kelletat, Andreas F.; Krumm, Hans-Jürgen; Krusche, Dietrich; Bohrer, Kurt-Friedrich (Dokumentation) (Hrsg.): Jahrbuch Deutsch als Fremdsprache, Band 21. Intercultural German Studies (Ottmar Hertkorn)

Wildner, Paul Peter (Hrsg.): Deutschunterricht in Österreich. Versuch eines Überblicks (Harald Tanzer)

Wolf, Volker (Hrsg.): Lesen und Schreiben. Literatur - Kritik - Germanistik. Festschrift für Manfred Jurgensen (Rüdiger Heßling)

Zentrum für Türkeistudien (Hrsg.): Das Bild der Ausländer in der Öffentlichkeit. Eine theoretische und empirische Analyse zur Fremdenfeindlichkeit (Matthias Grünewald)

Zima, Peter V. (Hrsg.): Literatur intermedial. Musik, Malerei, Photographie, Film (Thomas Bleicher)

$2 / 3,414-416$

$2 / 3,416-419$

$2 / 3,419-420$

$2 / 3,421-424$

$5,738-740$

$2 / 3,426$

2/3, 426-429

$2 / 3,429-431$

$2 / 3,431-433$

2/3, 433-435

$2 / 3,435-439$

$2 / 3,424-425$

$2 / 3,439-440$

$2 / 3,440-441$

$2 / 3,441-444$

$2 / 3,444-446$

$2 / 3,446-449$

$2 / 3,449-453$

$2 / 3,453-455$

\section{Stichwortregister}

Arbeitsgemeinschaften der Direktoren der Studienkollegs 4, 545-549 
Auslandsstudium finnischer Wirtschaftsstudenten 6,818-828

Auswahlbibliographie von Neuerscheinungen DaF 1996 1, 131-163

berufsbezogen studieren (Germanistikstudium in Nigeria) $1,58-68$

brasilianische Hochschulen (Deutschstudien) 4, 493-498

Bukarest 5, 656-660

Bulgarien 5, 661-666

Curriculumplanung (Thesen) 4, 470-479

Das Eigene und das Fremde sehen (Stadtführer Bukarest) 5, 656-660

DDR und Deutsch als Fremdsprache 6, 780795

Deutsch als fremde Wissenschaftssprache 6, 757-770

Deutsch als Fremdsprache

Arbeitsgemeinschaften der Direktoren der Studienkollegs 4, 545-549

Auslandsstudium finnischer Wirtschaftsstudenten 6,818-828

Auswahlbibliographie von Neuerscheinungen DaF 1996 1, 131-163

Auswahlbibliographie von Neuerscheinungen DaF 1996 (kommentiert) 2/3, 169$455 ; 5,695-740$

berufsbezogen studieren (Germanistikstudium in Nigeria) $1,58-68$

brasilianische Hochschulen 4, 493-498

Bukarest 5, 656-660

Curriculumplanung 4,470-479

Das Eigene und das Fremde sehen (Stadtführer Bukarest) 5, 656-660

in der DDR 6, 780-795

Deutsch als fremde Wissenschaftssprache $6,757-770$

deutsch-peruanische Begegnungsschule 6, 808-817

Deutsche Schulen im Ausland 6, 808-817

Deutschlehrerausbildung 4, 470-479

und Landeskunde 4,499-513

DSH-Fachtagung 1996 in Regensburg 1, 114-122

Einwanderungsdiskussion (Tagungsankündigung der DFG) 1, 127-129

Emotionen im DaF-Unterricht 5, 587-606

Erwachsenenalter und Fremdsprachenlernen $1,69-77$

Etymologisierung im Fremdsprachenunterricht 1,20-24

Fachsprache Wirtschaft (HRK-Zertifikat) $4,530-541$
Fachverband Deutsch als Fremdsprache 1, 114-122; 5, 559-586; 5, 741-743

»Falsche Freunde « 5, 661-666

Fernstudiengang für DaF-Lehrer an mexikanischen Hochschulen 4,480-492

finnische Wirtschaftsstudenten im Ausland 6, 818-828

Fremdsprachen lehren lernen (Tagungsankündigung der DGFF) 1, 126-127

Fremdsprachenlernen im höheren Erwachsenenalter 1, 69-77

Fremdverstehen durch FSU 1, 78-86

Für Sie gelesen (Kommentare und Rezensionen von Neuerscheinungen DaF 1996) $2 / 3,169-455 ; 5,695-740$

Germanistik in der Türkei 5, 621-624

grammatikalische Beschreibung 1, 25-50

HRK-Zertifikat »Fachsprache Wirtschaft» $4,530-541$

Hypertext und Fremdsprachenlernen in USA 5, 607-620

Hypermedia und Fremdsprachenlernen in USA 5, 607-620

interkulturelles Lernen 1,78-86

Internationalismengebrauch deutschlernender Bulgaren 5, 661-666

Jahrestagung DaF 1998 in Jena (Vorankündigung) 5, 741-743

Kabarett-Texte im DaF-Unterricht $\quad$ 1,87-98

Kommentierte Auswahlbibliographie von Neuerscheinungen DaF 1996 2/3, 169$455 ; 5,695-740$

in Korea (Tagungsbericht) 4, 542-544

Korea-Sprachpraxis in der Germanistik 5, 640-651

Kulturkontrast und Hermeneutik 6, 796807

Landeskunde in der Deutschlehrerausbildung 4,499513

in Ostasien 1,99-113

Videobeiträge im DaF-Unterricht (Jugendmagazin TURBO) 5, 652-655

Lehrer an mexikanischen Hochschulen 4, 480-492

Lehrerausbildung in der Diskussion (Tagungsankündigung der DGFF) 1, 126127

Lehrwerk für Thailand 1,51-57

Lernergrammatik 1,25-50

mexikanische Hochschulen (Fernstudiengang für DaF-Lehrer) 4, 480-492

Migration (Tagungsankündigung der DFG) $1,127-129$ 
Nigeria (Germanistikstudium) 1, 58-68

Peru 6, 808-817

Presseerklärung der Arbeitsgemeinschaften der Direktoren der Studienkollegs 4, 545-546

Oybiner Erklärung zur Rolle und zum Stellenwert der Studienkollegs 4, 547-549

Partizipialattribut 6,771-779

Polen (Stereotypenbilder über Deutschland und Deutsche) 5, 625-639

Rechenschaftsbericht des FaDaF für 1996/ 97 5, 667-672

Rügener Erklärung zur Rolle und zum Stellenwert der Studienkollegs 4,547

Sprachgeragogik 1,69-77

Stadtführer Bukarest 5, 656-660

Stereotypenbilder über Deutschland und Deutsche in Polen 5, 625-639

Studienstandort Deutschland

Deutsch als Fremdsprache und der 5, 559-586

die Rolle der Studienkollegs 4, 545-549

Studierende und Wissenschaftler 6, 751756

Textfunktion und Textstruktur 4, 514-522

Textkompetenz 4,514-522

Textproduktion 1,3-19

Textsorte Kabarett-Texte im DaF-Unterricht $1,87-98$

Textstruktur und Textfunktion $\quad 4,514-522$

Türkei 5, 621-624

USA (Videos im DaF-Unterricht)

Veranstaltungskalender 1, 123-125

Videobeiträge im DaF-Unterricht (Jugendmagazin TURBO) 5, 652-655

Videos im DaF-Unterricht in USA

Wirtschaftsstudenten im Ausland 6, 818828

Wissenschaftler und Studierende 6, 751756

Wörterbuch und Textproduktion 1,3-19

deutsch-peruanische Begegnungsschule 6, 808-817

Deutsche Schulen im Ausland 6, 808-817

Deutschlehrerausbildung 4,470-479

Deutschlehrerausbildung und Landeskunde 4, 499-513

Deutschstudien an brasilianischen Hochschulen 4, 493-498

DFG 1, 127-12129

DGFF 1, 126-127

DSH-Fachtagung I in Regensburg 1, 114-122

DSH-Fachtagung II in Regensburg 6, 829-843
Einwanderungsdiskussion (Tagungsankündigung der DFG) 1, 127-129

Emotionen im DaF-Unterricht 5, 587-606

Erwachsenenalter und Fremdsprachenlernen $1,69-77$

Etymologisierung im Fremdsprachenunterricht 1,20-24

Europa und Fremdsprachenpolitik 4, 459469

»Fachsprache Wirtschaft « (HRK-Zertifikat) $4,530-541$

Fachtagungen

Umsetzung der neuen DSH-Ordnung in die Prüfungspraxis 1, 114-122

Die DSH in der Praxis: Prüfungsvorbereitung 6, 829-843

Fachverband Deutsch als Fremdsprache 1, 114-122; 5, 559-586; 5, 741-743

»Falsche Freunde« 5, 661-666

Fernstudiengang für DaF-Lehrer an mexikanischen Hochschulen 4, 480-492

finnische Wirtschaftsstudenten im Ausland $6,818-828$

Fremdsprachen lehren lernen (Tagungsankündigung der DGFF) 1, 126-127

Fremdsprachenlernen im höheren Erwachsenenalter $1,69-77$

Fremdsprachenunterricht

Etymologisierung im Fremdsprachenunterricht 1,20-24

Fremdverstehen durch FSU 1, 78-86

und Sprachenpolitik im vereinten Europa 4, 459-469

Für Sie gelesen (Kommentare und Rezensionen von Neuerscheinungen DaF 1996 2/3, $169-455 ; 5,695-740$

GAL 1, 129-130

Germanistik in der Türkei 5, 621-624

Germanistikstudium und Arbeitsmarkt in Nigeria 1,58-68

grammatikalische Beschreibung 1,25-50

HRK-Zertifikat »Fachsprache Wirtschaft« 4, 530-541

Hypertext und Fremdsprachenlernen in USA 5, 607-620

Hypermedia und Fremdsprachenlernen in USA 5, 606-620

interkulturelles Lernen 1,78-86

Internationalismengebrauch deutschlernender Bulgaren 5, 661-666

Jena (Jahrestagung DaF 1998 - Vorankündigung) 5, 741-743

Kabarett-Texte im DaF-Unterricht $\quad 1,87-98$ 
Kommentierte Auswahlbibliographie von Neuerscheinungen DaF 1996 2/3,169-455; $5,695-740$

Korea - DaF in (Tagungsbericht) 4, 542-544 Korea-Sprachpraxis in der Germanistik 5, 640-651

Kulturkontrast und Hermeneutik 6, 796-807

Landeskunde in der Deutschlehrerausbildung 4,499-513 in Ostasien 1,99-113

Stereotypenbilder über Deutschland und Deutsche in Polen 5, 625-639

Lehrer an mexikanischen Hochschulen 4, 480-492

Lehrerausbildung in der Diskussion (Tagungsankündigung der DGFF) 1, 126-127

Lehrwerke

Aufgabenstellung in einem thailandspezifischen Lehrwerk 1,51-57

thailandspezifisches Deutschlehrwerk 1, 51-57

Lernergrammatik 1,25-50

mexikanische Hochschulen (Fernstudiengang für DaF-Lehrer) 4, 480-492

Mehrsprachigkeit in Europa 4, 544-545

Migration (Tagungsankündigung der DFG) $1,127-129$

Mitgliederversammlung des FaDaF 1997 (Protokoll) 5, 673-675

Nigeria (Germanistikstudium und Arbeitsmarkt 1,58-68

Oybiner Erklärung zur Rolle und zum Stellenwert der Studienkollegs 4,547-549

Partizipialattribut 6, 771-779

Peru 6, 808-817

Polen (Stereotypenbilder über Deutschland und Deutsche) 5, 625-639

Rechenschaftsbericht des FaDaF für 1996/97 5, 667-672

Regensburg (Fachtagung DSH I, 1996) 1, 114122

Regensburg (Fachtagung DSH II, 1997) 6, 829-843

Rügener Erklärung zur Rolle und zum Stellenwert der Studienkollegs 4,547

Sprachgeragogik 1,69-77

Sprachenpolitik und Fremdsprachenunterricht im vereinten Europa 4, 459-469

Sprachpraxis in der Germanistik in Korea 5, 640-651

Stadtführer Bukarest 5, 656-660

Stereotypenbilder über Deutschland und Deutsche in Polen 5, 625-639

Studienkollegs 4,545-549
Studienkollegs und ihr Stellenwert für den Studienstandort Deutschland 4,545-549

Studienstandort Deutschland und Deutsch als Fremdsprache 5, 559-586

Studienstandort Deutschland und die Rolle der Studienkollegs 4, 545-549

Studierende und Wissenschaftler und DaF 6, 751-756

Tagungsankündigungen

DFG: 2. Düsseldorfer Arbeitstagung im Rahmen des DFG-Projektes »Einwanderungsdiskussion im internationalen Vergleich « vom 19. bis 20.2.1998 1, 127-129

DGFF: 17. Kongreß für Fremdsprachendidaktik der Deutschen Gesellschaft für Fremdsprachenforschung (DGFF) vom 6.-8.10.1997 an der Universität KoblenzLandau 1, 126-127

FaDaF: Vorankündigung der 26. Jahrestagung Deutsch als Fremdsprache des Fachverbandes Deutsch als Fremdsprache vom 4. bis 6. Juni 1998 an der Friedrich-Schiller-Universität Jena $5,741-743$

GAL: 28. Jahrestagung der Gesellschaft für angewandte Linguistik (GAL) e. V. vom 25.-27.9.1997 an der Universität Bielefeld $1,129-130$

Tagungsberichte

Deutsch als Fremdsprache in Korea 4, 542544

Die chinesische Germanistik im 21. Jahrhundert 6, 844-849

Fachtagung zur DSH-Rahmenordnung I 1, 114-122

Fachtagung zur DSH-Rahmenordnung II $6,829-843$

17. Frühjahrskonferenz zur Erforschung des Fremdsprachenunterrichts zur Fremdsprachenlehrerausbildung und zum Fremdsprachenunterricht an Hochschulen (Erklärung zum Thema Mehrsprachigkeit in Europa) 4, 544-545

25. Jahrestagung des $\mathrm{FaDaF}$ in Mainz 5, 677-688

Textfunktion und Textstruktur 4, 514-522

Textkompetenz $\quad 4,514-522$

Textsorte Kabarett-Texte im DaF-Unterricht $1,87-98$

Textstruktur und Textfunktion $\quad 4,514-522$

Thailand 1,51-57

Türkei (Germanistik und DaF in der Türkei) 5, 621-624

USA (Hypertext und Hypermedia im Fremdsprachenunterricht) 5, 607-620 
USA (Videos im DaF-Unterricht) 4, 523-529

Videos im DaF-Unterricht in USA 4, 523-529

Videomagazin TURBO 5, 652-655

Veranstaltungskalender 1,123-127

vereintes Europa und Sprachenpolitik 4, 459-469

Wissenschaftler und Studierende und DaF 6, 751-756

\section{Autorenregister}

Aguado, Karin 5, 733-738

Albers, Hans-Georg 5, 684-687

Althoff, Roswitha 2/3, 284-288

Altmayer, Claus $2 / 3,213-215 ; 237-241 ; 292-$ 296

Ansorge, Monica 2/3, 364-365

Balcı, Tahir 5, 621-624

Barner, Axel 5, 656-660

Bayerlein, Oliver 2/3, 193-194; 288-290

Berndt, Annette 1,69-77

Bettermann, Rainer 5, 689-694

Bhatti-Küppers, Heidemarie 2/3, 169-455; 5, 655-740

Bickes, Gerhard 2/3, 282-284

Blei, Dagmar 6, 780-795

Bleicher, Thomas 2/3, 367-369; 453-455

Blühdorn, Hardarik 2/3, 257-262

Bode, Christian 6,751-756

Bohle, Kirsten 2/3, 358-360

Bolten, Jürgen $\quad 4,530-541$

Borowsky, Viktor A. 1, 123-125

Bosch, Gloria 4, 459-469

Bräsel, Sylvia 1,99-113; 5, 704-706; 5, 725-728

Braun, Christiane 5, 680-683

Dannerer, Monika 2/3, 262-264

Dietz, Gunther 2/3, 319-323; 345-349

Doval, Irene $\quad 2 / 3,435-439$

Dreischer, Anita 2/3, 388-391

Drewnowska-Vargáné, Ewa 4, 514-522

Eggensperger, Klaus 4,493-498

Eggers, Dietrich 1,131-163; 5, 559-586

Ehlich, Konrad 6,757-770

Ehnert, Rolf 5, 559-586

Engel, Henrik 2/3, 179-184; 328-333; 351355; 381-384

Esselborn, Karl 2/3, 189-193; 323-326, 369372

Fandrych, Christian

Florin, Karl-Walter

$4,480-492$

384-385

Freyer-Wojnikowa

2/3, 224-225; 246-248;

$2 / 3,439-440 ; 440-441$
Vorstand des FaDaF 1997-1999 5, 675-677

Wirtschaftsstudenten im Ausland 6, 818-828

Wissenschaftler und Studierende und DaF 6, 751-756

, Zertifikat »Fachsprache Wirtschaft « 4, 530541

Götze, Lutz 2/3, 356-358

Groß, Michael 2/3, 216-218; 279-281; 349351

Grünewald, Matthias 2/3, 249-251; 426-429; 449-453

Harnischfeger, Johannes $1,58-68$

Hartmann, Daniela 2/3, 200-204; 305-308; 416-419

Hartmann, Ralph 2/3, 198-200; 362-363

Heinecke, Ilse 2/3, 308-309; 408; 426

Henkenborg-Schröder, Renate 5, 687-688

Hertkorn, Ottmar 2/3, 441-444

Heßling, Rüdiger $2 / 3,386-388 ; 446-449$

Hilker, Claudia $\quad 2 / 3,194-198 ; 385-386$

Hinrichs, Beatrix $\quad 6,808-817$

Honnef-Becker, Irmgard 2/3, 339-341

Jahnel, Andrea 2/3, 251-255; 273-277; 393397

Jankowski, Peter 2/3, 403-405

Jaumann, Peter 2/3, 266-269

Kaikkonen, Pauli 1,78-86

Kim, Hi-Youl 5, 640-651

Kirsch, Klaus 5, 559-586

Krekeler, Christian 2/3,408-411

Königs, Frank G. 5, 677-680

Köppel, Rolf $\quad 6,844-849$

Köster, Lutz 2/3, 187-189

Krefting, Brigitte 5, 673-675; 684-687

Latour, Bernd $2 / 3,277-278 ; 392-393 ; 419-420$

López Barrios, Mario 1,3-19

Luchtenberg, Sigrid 2/3, 341-343

Majari, Chris F. 5, 607-620

Meuter, Jörg 5, 652-655

Mikova, Katarina 2/3, 374-375

Mitschian, Haymo 2/3, 397-399; 414-416

Mohácsi, János 5, 722-723

Mohr, Inge 2/3, 365-367; 433-435

Müller-Küppers, Evelyn 1, 131-163; 2/3, 169-455; 5, 695-740

Neuf-Münkel, Gabriele 5, 677-680

Niedenthal, Carola $\quad 2 / 3,314-319$ 
Nord, Holger $\quad 2 / 3,225-227$; 336-339

Otto, Detlev 2/3, 411-414

Padberg, Gabriele 2/3, 355-356

Reinbothe, Roswitha 4, 499-513

Reuter, Ewald 2/3,311-314

Richter, Regina $\quad 2 / 3,185-187 ; 290-292$

Riechert, Rüdiger $2 / 3,178 ; 372-373$

Riecke, Jörg 2/3, 205-208

Rösch, Gertrud Maria 2/3, 241-246; 270-272; 309-311

Rößer, Hans-Otto 2/3, 333-336

Roggausch, Werner 4, 470-479; 6, 796-707

Saarbeck, Ursula 2/3, 269-270; 327-328

Saengaramruang, Wanna 1,51-57

Sandner, Arno 1, 131-163

Schlabbach, Joachim $\quad 6,818-828$

Schleyer, Walter 5, 699-704; 711-715

Schmidt, Georg 5, 738-740

Schreiber, Rüdiger 5, 680-683

Schütz, Sonja 1, 87-98

Schuster, Britt-Marie 2/3, 233-236

Schwarck, Dorothee 1,131-163

Schwerdtfeger, Inge Christine 5, 587-606

Seifert, Jutta 2/3, 405-406

Siebke, Martina 1, 20-24

Sölter, Arpad 2/3, 209-211

Sonntag, Stefani 2/3, 375-376

Stephani, Christiane 6,771-779

Stein-Meintker, Anneliese 2/3, 400-403; 5, 706-709

Stief, Christine 2/3, 231-233
Stöver-Blahak, Anke 5, 677-680

Tallowitz, Ulrike $\quad 4,480-492$

Tanzer, Harald $\quad 2 / 3$, 255-257; 264-266; 444446

Topalova, Antoanita 5, 661-666

Tröml, Sibille 2/3, 302-305; 378-381

Unterbäumen, Enrique Huelva 1, 25-50; 5, 716-719

Vinkalne, Ieva 2/3, 407-408

Volmer, Astrid 5, 719-722

Vorderwülbecke, Klaus 5, 680-683

Wagner, Andrea 2/3,360-362

Waumans, Wim 2/3, 218-222; 429-431

Wehage, Franz-Joseph 4, 523-529

Weigt, Zenon 5, 728-731

Wiesmann, Bettina 1, 114-122; 2/3, 227-229; $6,829-843$

Wilde-Stockmeyer, Marlis 5, 715-716; 724725

Wildegans, Gotthard 2/3, 421-424; 431-433

Willkop, Eva-Maria 2/3, 297-301; 5, 695-699

Wintermann, Bernd 5,667-672; 684-687

Wolff, Armin 1, 123-125; 5, 559-586; 6, 854866

Wolfsteiner, Beate $2 / 3,296-297 ; 377-378$

Wolter, Inula $5,709-711$

Wüst, Inge $2 / 3,223-224 ; 343-345 ; 424-425$

Yendell, Maria $2 / 3,212-213 ; 5,731-733$

Zimmer, Thomas 4, 542-544

Żurek, Jolanta 5, 625-639

(zusammengestellt von Armin Wolff, Regensburg) 